\title{
Continuing medical education (CME) for primary care health professionals
}

The revised BTS/SIGN guideline [1] makes a grade $B$ recommendation that all people with asthma should have access to primary care delivered by clinicians with appropriate training in asthma management. This is a laudible recommendation; however, the impact of much of the continuing medical education (CME) for primary care health professionals on managing patients with asthma remains unclear. Looking at the systematic review published in this edition of the Journal, it would appear that there is little substantive evidence to support this casual relationship, which if true brings into questions much of our current educational intervention. However, intuitivelyeit would be assumed that training heatthprofessionals would enable them to acquire new skills and information, and as a consequence patient outcomes would be improved.

The systematic review paper by Barton et al. (2004) [2] concludes that following a review of the published randomised controlled trials, that no recommendations can be made on the effectiveness of CME for improving health outcomes of patients with asthma. However it should be noted that only three studies met their inclusion criteria, so the review may have some limitations in practice. The review by Barton and colleagues highlights several issues associated with developing a body of evidence in this field. One key issue is the methodologies used. Reviewing solely randomised controlled trials ignores other methodologies more frequently accepted within the field of educational research, which may be more appropriate.

A meta-analysis by Davis et al. [3] which looked more broadly at studies that objectively assessed physician performance and/or health outcomes showed more positive outcomes. 99 trials, containing 160 interventions were reviewed. 101 of 160 interventions displayed an improvement in at least one major outcome measure; $70 \%$ demonstrated a change in physician performance and $48 \%$ aimed at health outcomes produced a positive change.
Because of the multifaceted influences on patient behaviour it is difficult to attribute change in patient outcomes to direct exposure to individual health professional education. Any reduction in symptoms and improvements in quality of life is likely to depend on many patient related factors including knowledge of their disease, appropriateness and effectiveness of treatment, adherence to therapy regimes and regular follow up. Patients increasingly have various sources of health information in addition to that acquired from health professionals, such as friends and family, patient (literature and increasingly the internet.

There are also other external influences on improved patient outcomes other than education of health professionals and patient behaviour. Between 1988 and 1998 the death rate from asthma in the UK dropped from 2000 to 1500 . During this time specialist training for health professionals in primary care in asthma management was widely introduced. However during this decade many other changes and interventions happened in the field of asthma. These included the introduction of the British Thoracic Society (BTS) guidelines in asthma management, whereby the government introduced an enhanced role for primary care with financial incentives to improve chronic disease management including asthma care; a wide range of new pharmaceuticals and increased recognition and use of the practice nurse's role in asthma management.

Several studies during that time attempted to evaluate improved patient outcomes such as decreased frequency of symptoms, number of acute attacks and fewer days lost from school/work as a result of formal accredited training of practice nurses [4-6]. Although there were notable improvements across all three domains it was difficult to claim that the improvements occurred solely due to the training.

The assessment of the impact of such a broad term as CME is in itself problematical as CME 
encompass a wide range of interventions from didactic lectures to small group problem based learning, to on-line intrapersonal learning. The outcomes of any education will only be as good as the educational intervention itself. Even if transportation of knowledge occurs as a result, this may not necessarily improve patient outcomes or practitioner behaviour.

The work of Clark and colleagues [7] from Michigan University specifically addresses not only the what, but the how to get effective messages across to patients in their physician education programmes. The how is an area that is frequently overlooked in many CME programmes in the belief that health professionals already are effective communicators having gained this knowledge by experience or previous training. Greater concentration on this in educational programmes, may be the real key to improving patient outcomes.

It will always be a problematical area to research, although this should not prevent those of us with an interest in the fields of education and research from identifying ways of so doing. Educational interventions for healthcare professionals represent one strategy for improving patient outcomes. The National Respiratory Training Centre (NRTC), which is an educational institution, is in the process of evaluating the outcomes of its health professional training programmes on patients as the true effectiveness of such training is las yet unknown.

The study is a multi-centre community-based parallel group randomised controlled trial comparing the effects of the NRTC allergy course for primary care professionals with usual care on patients with perennial rhinitis. 202 patients have been randomised to receive either care from an allergy-trained health professional or usual care from a GP or nurse who has not received training. The primary outcome is the effect on disease-specific quality of life measured by the mini-Rhinoconjunctivitis Quality of Life questionnaire [7] 6 months after completion of the health professional training programme. Secondary outcome measures include differences in Asthma Quality of Life Questionnaire (AQLQ) scores, number of primary care consultations for rhinitis, and proportion of patients in whom allergy diagnostic skin prick tests were performed.

This is an ambitious study which may lead us to evaluate educational programmes more effectively in the future.

With the lack of definitive evidence of the benefit of health professional CME on patient outcomes, persevering with it continues to be a leap of faith. However as the authors of the published paper state: 'absence of proof is not the same as proof of absence'. Educationalists and researchers owe it to both clinicians and patients to continue to develop the evidence in this neglected area in order to establish the most effective interventions.

\section{References}

[1] 2004 Update Guideline Download from BTS/SIGN The BTS/SIGN British Guideline on the Management of Asthma.

[2] Continuing medical education for asthma in primary care settings: a review of randomised controlled trials. Christopher Barton, Nabil Sulaiman, Siaw-Teng Liaw Prim Care Resp J 2003 12(4):119-23. http://www.gpiag.org/journ/ vol12_4/119_123barton.pdf.

[3] Davis DA, Thomson MA, Oxman AD, Haynes RB. Changing physician performance. A systematic review of the effect of continuing medical education strategies, 1. JAMA 1995;274(9):700-5

[4] Hoskins et al. The tink between practice nurse training and asthma outcomes. Brit J Community Nursing 1999;4:222-28.

[5] Juniper EF, Thompson AK, Ferrie PJ, Roberts JN. Development and validation of the Mini Rhinoconjunctivitis Quality of Life Questionnaire. Clinical and Experimental Allergy 2000;30:132-40.

[6] Neville RG, Hoskins G, Smith B, Clark RA. Observations on the structure, process and clinical outcomes of asthma care in general practice. Brit J Gen Pract 1996;46:583-7.

[7] Cabana MD, Clark N. Challenges in evaluating methods to improve physician practice. J Pediatr 2003 Oct;143(4): 413-4.

Monica Fletcher

The National Respiratory Training Centre (NRTC)

The Athenaeum, 10 Church Street Warwick CV34 4AB, England, UK

Tel.: +44 1926 493313; fax: +441926 493224

E-mail address: m.fletcher@nrtc.org.uk (M. Fletcher)

22 February 2004

Available online at www.sciencedirect.com

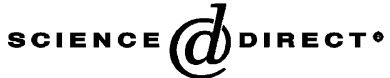

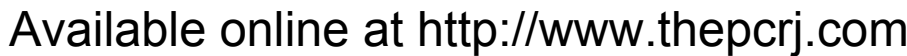

